\title{
Which post-marketing database and studies does EMEA require
}

\author{
Gottfried Kreutz, Leng Heng
}

Federal Institute for Drugs and Medical Devices, Department Clinical Pharmacology I,

Friedrich-Ebert-Allee 38, D-53113 Bonn, Germany

Correspondence to: Dr Kreutz
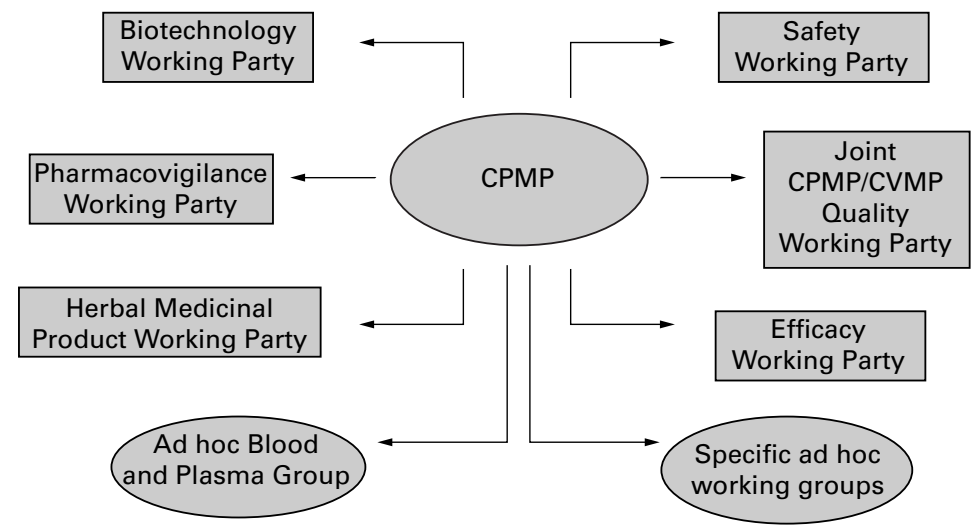

Figure 1 The Committee on Proprietary Medicinal Products (CPMP) and its Working Parties.

Initiation of the concept papers, points to consider and notes for guidance

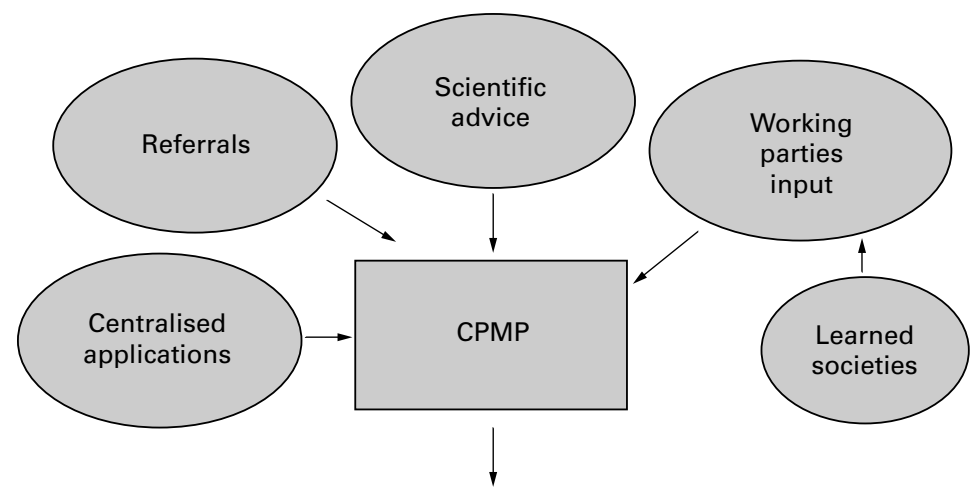

Concept papers, points to consider and notes for guidance

Figure 2 Initiation of Guidance Documents. ing ample opportunities for exchange of expertise and consensus discussions (fig 1).

Medical regulatory requirements including clinical efficacy and clinical safety aspects are discussed and drafted within the Efficacy Working Party. This work is resulting in Concept Papers, Points to Consider, Notes for Guidance, or Scientific Advice to specific questions asked by applicants (fig 2).

The objectives of these guidance documents are:

- to give guidance to pharmaceutical companies in planning the clinical development of new compounds to demonstrate therapeutic efficacy and clinical safety,

- to harmonise the requirements among assessors and experts of the different European National Regulatory Authorities in assessing data and documents,

- to adjust regulatory requirements to the advances in the biomedical domain,

- to contribute to the activities of the International Conference of Harmonization (ICH).

It is important to realise that the work process is characterised by networking and by openness. The network is shown in figure 3 and the process exemplified by showing steps for drafting efficacy guidance papers (fig 4).

All kinds of expert knowledge, regulatory, medical schools, learned societies, patient care, hospital or ambulatory are getting chances to become included. Important interfaces to achieve this are provided by National Regulatory Authorities and by supranational Learned Societies.

Decisions taken on the national level and within national procedures, which are still existing, normally have to take into consideration all aspects agreed upon on the European level. Here national bodies have important tasks to fulfill, too.

At this stage of development of procedures and regulatory requirements in the European Union there are no guidance papers specifically on post-approval therapeutic aspects other than adverse drug reaction reporting procedures and evaluation of case reports. But resulting from legal requirements it is clear that additionally to guidance covering preapproval and post-approval issues altogether new aspects resulting from application experience have to be considered and documentation and evaluation have to become supplemented accordingly. The new aspects that should earn specific attention from manufacturers, medical doctors, pharmacists, and patients are especially those that could not be studied sufficiently in the pre-approval period. 


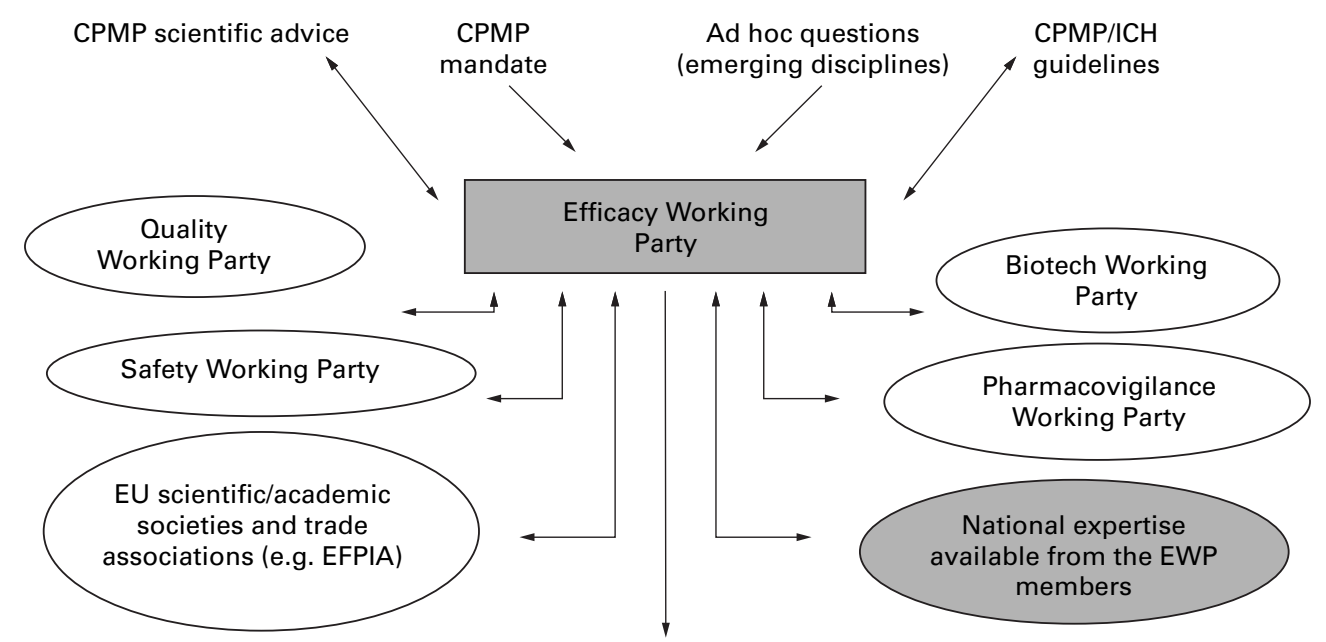

Specific ad hoc working groups or subgroup meetings when needed

European list of $>2000$ experts

Figure 3 Efficacy Working Party (EWP) and its Network.

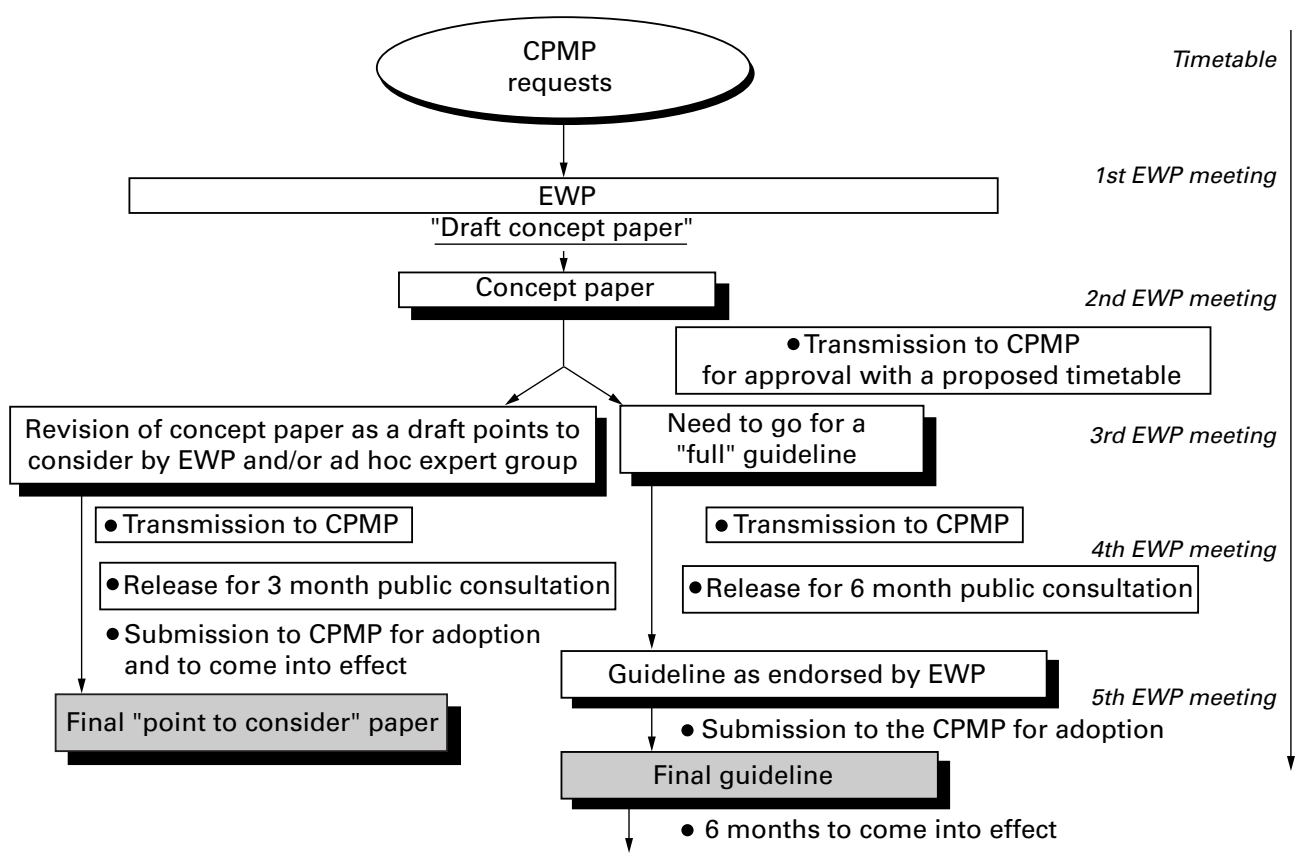

Figure 4 Steps for Drafting EWP Guidance Papers.

These are effects in patient populations not studied or studied only incompletely before approval, long term effects, interactions with exposure to other medication or nutrition, individuals or subpopulations identified as unexpectedly susceptible to specific risks or reduced efficacy.
Following these general rules there should be no major problems for rheumatologists to observe patients according to their needs and taking into consideration specific aspects of these diseases such as chronicity and seriousness on the one hand and potency of many of the newly developed treatments on the other. 